\title{
Role of ultrasound in evaluation of pharyngeal dysphagia in children with cerebral palsy
}

\author{
Ahmed S. Abdelrahman ${ }^{1 *}$, Emad H. Abdeldayem', Samia Bassiouny ${ }^{2}$ and Hanan M. Elshoura ${ }^{3}$
}

\begin{abstract}
Background: The aim of this study was to evaluate the clinical reliability of ultrasound (US) examination using relative laryngeal movement of $40 \%$ as a cutoff point to diagnose pharyngeal abnormalities of swallowing using ultrasonographic examination in dysphagic cerebral palsy (CP) patients and comparing its results with a flexible fiberoptic endoscope.

Methods: Twenty-five cerebral palsy children suffering from clinical dysphagia were included in this study. The rest distance between the thyroid cartilage and the hyoid bone and the shortest distance between them during swallowing were measured by ultrasound, then the approximation distance and the percentage of relative laryngeal movement were calculated. All children also have been submitted for flexible fiberoptic endoscopy (FEES).

Results: The mean value of the percentage of relative laryngeal movement was significantly less in the CP children with pharyngeal phase abnormality diagnosed by the flexible fiberoptic endoscope $(p<0.001)$. The mean of relative laryngeal movement in CP patients with and without pharyngeal abnormality diagnosed by the flexible fiberoptic endoscope was $20.10 \pm 13.73$ and $66.19 \% \pm 3.42$ respectively.

Conclusion: Ultrasound can efficiently measure the relative laryngeal movement, and as it gives a numerical value, it can be used as a follow-up bedside test in children suffering from dysphagia.
\end{abstract}

Keywords: Ultrasound (US), Cerebral palsy (CP)

\section{Background}

Cerebral palsy $(\mathrm{CP})$ is a chronic disorder affecting patient movement [1], and approximately $75 \%$ of $\mathrm{CP}$ children have dysphagia, which causes malnutrition and impacts their development [2].

The fiberoptic endoscopy (FEES) is used in the assessment of swallowing, particularly the pharyngeal stage [3]; it can be used to demonstrate the penetration and/or aspiration [4], but it does not provide direct information on laryngeal elevation or hyoid-larynx approximation [5].

Dynamic laryngeal movement during swallowing with laryngeal elevation and hyoid-larynx approximation is an essential component of swallowing [6,7], and it is associated with bolus passage through the pharynx,

\footnotetext{
*Correspondence: dr_ahmedsamy@yahoo.com

${ }^{1}$ Radiology Department, Faculty of Medicine, Ain Shams University, Cairo,

Egypt

Full list of author information is available at the end of the article
}

opening and closure of the cricopharyngeal muscle, and also airway protection $[8,9]$.

Ultrasound (US) can detect and recognize the hyoid bone and thyroid cartilage, and hence, the percentage of hyoid-laryngeal approximation can be calculated by the measurement of the approximation distances of the two structures [5].

The aim of this work is to assess the diagnostic performance of US for diagnosing abnormalities in the pharyngeal phase of swallowing in dysphagic $\mathrm{CP}$ patients using reduced relative laryngeal movement of $\leq 40 \%$ compared to flexible fiberoptic endoscopy.

\section{Methods \\ Patients}

The study was done in the Ain Shams Specialized Hospital during the period from August 2016 till February 2017. Twenty-five cerebral palsy children 
with dysphagia were included in this study. Both fiberoptic endoscopic examination and US examination were done in the same day. Written consents were taken from all parents of patients to participate in this study, according to the rules of the ethical committee.

\section{Technique of US examination}

- Ultrasound assessment was carried out by the same operator for all patients.

- Children sat with the assistance of their relative to the most comfortable position for them.

- An ultrasound was performed using an ultrasound machine (LOGIQ ${ }^{\text {ms }}$ P7, GE Healthcare) using a highfrequency probe $(7-10 \mathrm{MHz})$. Axial and sagittal ultrasound scanning was performed.

- The transducer was placed in the midsagittal plane, centered between the anterior border of the hyoid bone and the superior border of the thyroid cartilage, with the central sound wave running perpendicular to this plane.

- 2 to $3 \mathrm{ml}$ of water was inserted into patient's mouth, then the process of laryngeal elevation using dynamic images with the assist of a cine loop was evaluated (Fig. 1).

- The distance between the thyroid cartilage and hyoid bone before swallowing (rest distance) was measured and recorded as well as the shortest distance during the swallowing process (Fig. 2).

- The approximation distance was obtained by subtracting the shortest distance between the thyroid cartilage and hyoid bone during swallowing from the resting distance.

- Then the percentage of relative laryngeal movement was calculated by dividing the approximation distance by the resting distance.

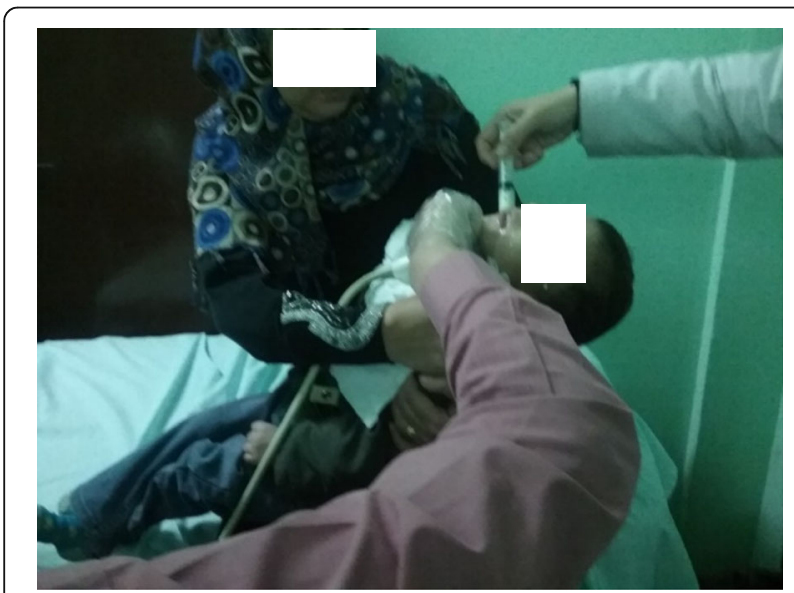

Fig. 1 Ultrasound evaluation of relative laryngeal movement
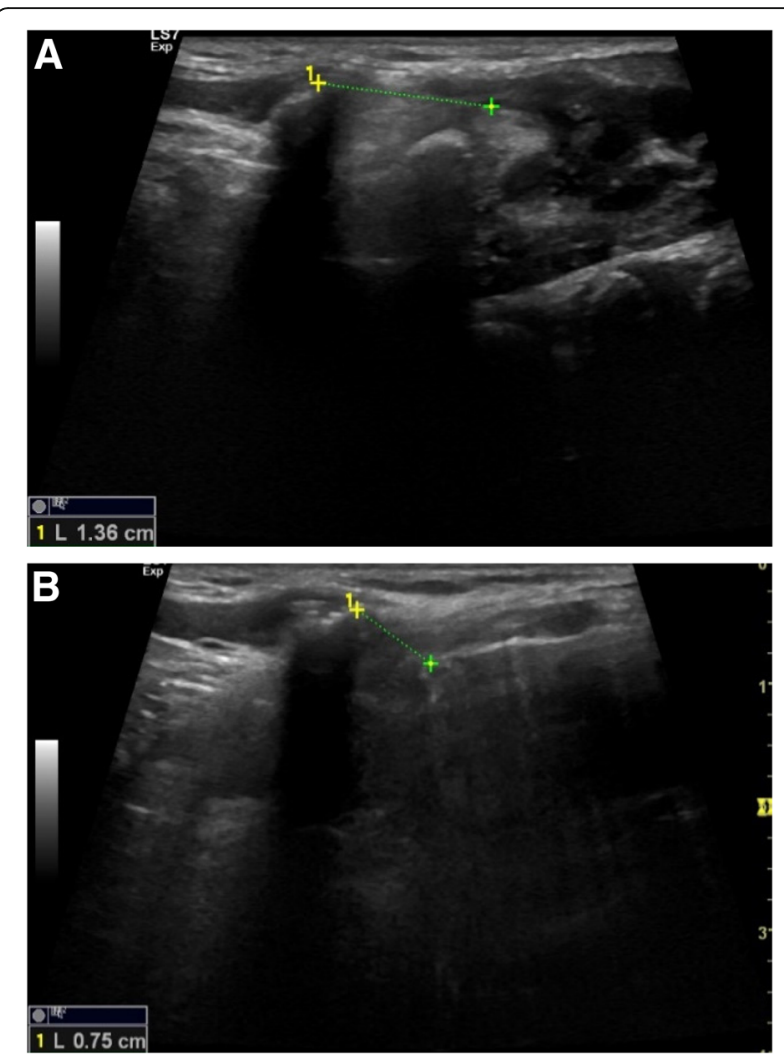

Fig. 2 Measurement of the thyroid cartilage hyoid bone distance. a The hyoid thyroid distance during rest. $\mathbf{b}$ The shortest hyoid thyroid distance during swallowing

- The swallowing process was repeated while the transducer was placed in the axial plane with sequential images obtained at the level of the thyroid cartilage to evaluate if there is spillage of water into the laryngeal airway (Fig. 3).

\section{Fiberoptic endoscopy (FEES)}

- FEES was carried out by a phoniatric to assess if there was a pharyngeal phase of swallowing abnormalities.

\section{Analysis of data}

- A relative laryngeal movement less than $40 \%$ [5] considered as a diagnostic value for the pharyngeal phase of dysphagia, and the US results were compared to the FEES results.

- The B mode examination was also done, and penetration/aspiration was diagnosed if water was seen at the level of the vocal cord.

- The analysis data was done using IBM SPSS statistics (V. 25.0, IBM Corp., USA, 2017). Data were expressed as mean \pm SD for quantitative 

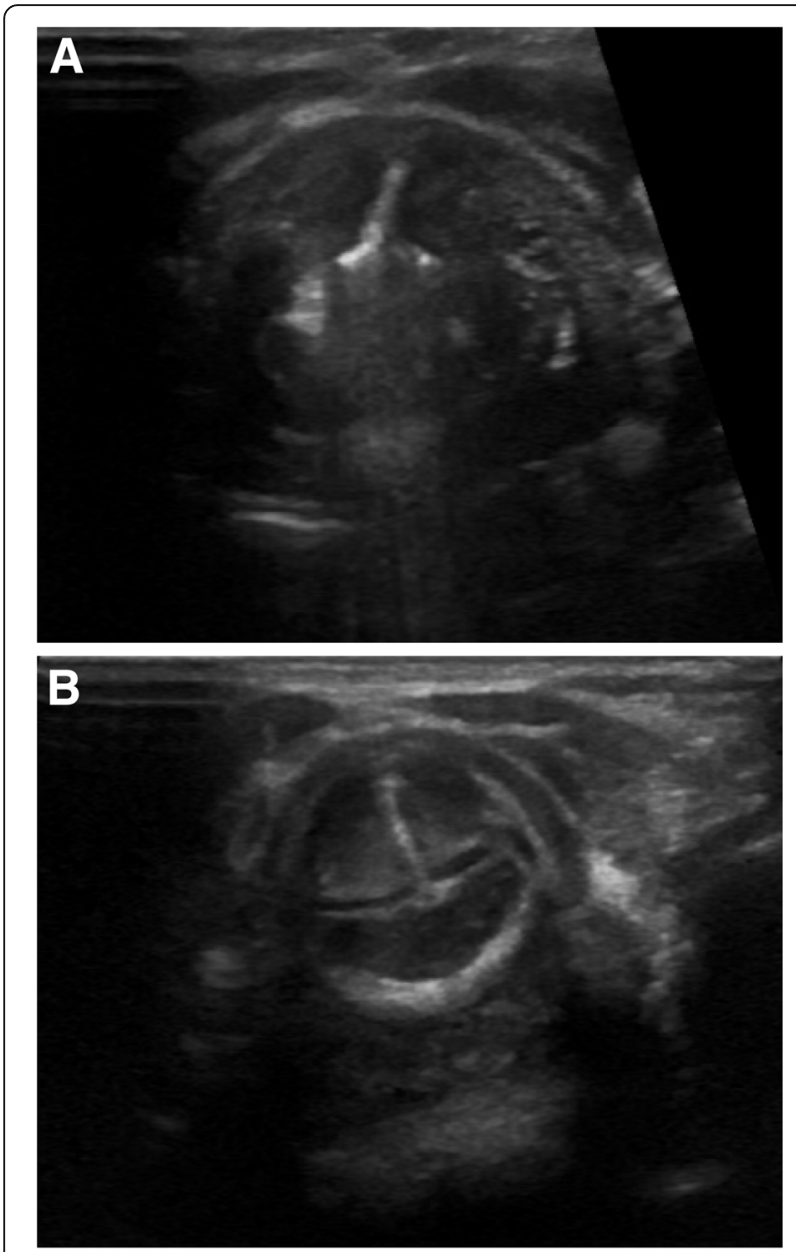

Fig. 3 B mode image at the level of the vocal cord with fine air bubble casting posterior reverberation artifact (a) and with no reverberation artifact $(\mathbf{b})$

parametric measures in addition to median percentiles for quantitative non-parametric measures and both the number and percentage of categorizing data. Diagnostic validity tests were used, including diagnostic sensitivity and specificity.

\section{Results}

Twenty-five CP children with a clinical diagnosis of dysphagia underwent both US examination and FEES. No adverse events occurred during both procedures. The median patient age was 3.5 years.

The median US resting distance was $1.39 \mathrm{~cm}$, and the median US shortest distance was $0.62 \mathrm{~cm}$, while the median US relative laryngeal movement was $63.28 \%$ (Table 1 ).

Of the 25 patients studied, the FEES examination revealed pharyngeal dysphagia in 10 out of $25(40 \%)$ patients, while the US examination revealed reduced relative laryngeal movement $(\leq 40 \%)$ in 9 out of $25(36 \%)$
Table 1 The median value measured by the US

\begin{tabular}{llll}
\hline US measurement & Median & Min. & Max. \\
\hline Rest distance & 1.39 & 0.83 & 2.48 \\
Shortest distance during swallowing & 0.62 & 0.23 & 1.8 \\
Percentage of relative laryngeal movement & $63.28 \%$ & $2 \%$ & $73.2 \%$ \\
\hline
\end{tabular}

patients with a significant correlation between both US and FEES ( $p$ value $<0.001$ ) (Table 2). The mean of the reduced relative laryngeal movement in these patients was $20.10 \pm 13.73$.

The FEES examination revealed normal swallowing in 15 out of $25(60 \%)$ patients while the US examination revealed normal relative laryngeal movement in 16 out of $25(64 \%)$ patients with a significant correlation between both US and FEES ( $p$ value $<0.001$ ). The mean of relative laryngeal movement in these patients was $66.19 \% \pm 3.42$. The false negative case shows borderline relative laryngeal movement of $45 \%$; yet during the $B$ mode examination, fluid was seen spilled into the laryngeal airway, and the final US diagnosis of this case was an abnormal pharyngeal phase of swallowing (Table 3).

The inter-method agreement between the reduced relative laryngeal movement $(\leq 40 \%)$ and FEES for diagnosing abnormalities of swallowing pharyngeal phase was $96 \%$, with sensitivity, specificity, positive predictor value, and negative predictor value of $90 \%, 100 \%, 100 \%$, and $93.75 \%$, respectively, and the cutoff of our study was $45 \%$ with $100 \%$ sensitivity, specificity, positive predictive value, and negative predictor value (Table 4).

\section{Discussion}

This study demonstrated that ultrasonography quantitatively evaluated reduced laryngeal movement help in the assessment and diagnosis of pharyngeal phase dysphagia in CP children with sensitivity of $96 \%$, and it can be used as an alternative to the FESS as it is a less-invasive and less costly bedside test.

Cerebral palsy is a permanent movement disorder which is attributed to disturbances in the developing fetal or infant brain, and it appears in early childhood [1].

Dysphagia is closely related to gross motor dysfunction in children with CP [10], and oropharyngeal dysphagia occurs in about $85 \%$ of children with CP [11].

Over the years, endoscopy has become a standard tool for diagnosing dysphagia [3] [12]. FEES investigates the pharyngeal phase only [13].

Real-time ultrasound is a non-ionizing imaging modality and has been used to observe deglutitive movements since the late 1970s [14, 15]. It was used in preliminary investigations of infants to visualize the bolus movement through the pharyngeal area while they are being breastfed [13]. 
Table 2 Pharyngeal dysphagia diagnosed by the reduced relative laryngeal movement $(\leq 40 \%)$ as compared to FEES which was used as the reference modality

\begin{tabular}{|c|c|c|c|c|c|}
\hline & & & Pharyn & sed by FEES & Total \\
\hline & & & Neg & Pos & \\
\hline Pharyngeal dysphagia diagnosed by & Neg & Count & 15 & 1 & 16 \\
\hline reduced relative laryngeal movement & & $\%$ & $100 \%$ & $10 \%$ & $64 \%$ \\
\hline & Pos & Count & 0 & 9 & 9 \\
\hline & & $\%$ & $0.0 \%$ & $90 \%$ & $36 \%$ \\
\hline Total & & Count & 15 & 10 & 25 \\
\hline & & $\%$ & $100 \%$ & $100 \%$ & $100 \%$ \\
\hline
\end{tabular}

Larynx elevation is crucial for airway protection and cricopharyngeal muscle relaxation $[9,16,17]$. As the thyroid cartilage approximates to the hyoid bone, the larynx elevates, the epiglottis tilts down and closes to prevent the bolus from entering the trachea, and cricopharyngeal muscle opens subsequently, allowing the bolus to pass through $[18,19]$. So incomplete laryngeal elevation is assumed to be one of the major mechanisms in dysphagia and often leads to aspiration [20]. The fiberoptic endoscopic evaluation of deglutition is invasive and does not allow one to quantify swallowing movements [21].

The relative laryngeal movement is used in this study rather than the approximation distance to exclude the normal interpersonal variation, especially as the study deals with children in different ages.

In this study, the mean relative laryngeal movement in CP children with no pharyngeal abnormalities was $66.19 \% \pm 3.42$. Similar results were concluded by Kuhl et all. [22] who have examined 42 healthy people and 18 dysphagic patients suffering from different neurological diseases, and they found that the mean relative laryngeal movement was $61 \% \pm 3$ in normal subjects. Huang et al. [5] have also examined 15 normal volunteers, 20 patients without dysphagia, and 20 patients with dysphagia, and they found that the mean relative laryngeal movement in normal volunteers was $47.26 \pm 4.9 \%$ and in stroke nondysphagic patients $42.66 \pm 8.3 \%$.

In our study, the mean relative laryngeal movement in the dysphagic CP children suffering from pharyngeal abnormalities was reduced to less than $40 \%(20.10 \pm 13.73)$; similar results were also concluded by Huang et al. [5] and Kuhl et al. [22] who showed reduced relative laryngeal movement to less than $40 \%$ in patient suffering from dysphagia ( $34.06 \pm 10.9 \%$ and $42 \% \pm 10$ respectively).

In addition to the evaluation of relative laryngeal movement, B mode examination at the level of the thyroid cartilage may increase the sensitivity of US examination in diagnosing the pharyngeal phase of dysphagia, as the spillage of water into the laryngeal airway could be visualized in these children as air bubbles intermixed with water droplets, and it was beneficial in this study as the only false negative case which showed borderline reduced relative laryngeal movement (45\%); penetration of water over the vocal cord was identified during $\mathrm{B}$ mode examination and the final US diagnosis was pharyngeal dysphagia.

The reduced relative laryngeal movement cut off value in this study was $45 \%$, which was also similar to Huang et al.'s [5] result who found that the relative laryngeal movement of less than $40 \%$ may imply dysphagia, with acceptable sensitivity and specificity.

Table 3 Pharyngeal dysphagia diagnosed by US, including both the reduced relative laryngeal movement ( $\leq 40 \%)$ and the B mode as compared to FEES which was used as the reference modality

\begin{tabular}{|c|c|c|c|c|c|}
\hline & & & \multicolumn{2}{|c|}{$\begin{array}{l}\text { Pharyngeal dysphagia diagnosed by reduced relative laryngeal movement and B } \\
\text { mode }\end{array}$} & \multirow[t]{2}{*}{ Total } \\
\hline & & & $\overline{\mathrm{Neg}}$ & Pos & \\
\hline \multirow[t]{4}{*}{ Pharyngeal dysphagia diagnosed by FEES } & Neg & Count & 15 & 0 & 15 \\
\hline & & $\%$ & $100 \%$ & $0.0 \%$ & $60 \%$ \\
\hline & Pos & Count & 0 & 10 & 10 \\
\hline & & $\%$ & $0.0 \%$ & $100 \%$ & $40 \%$ \\
\hline \multirow[t]{2}{*}{ Total } & & Count & 15 & 10 & 25 \\
\hline & & $\%$ & $100 \%$ & $100 \%$ & $100 \%$ \\
\hline
\end{tabular}


Table 4 The diagnostic validity of reduced laryngeal movement of $\leq 40 \%$ for diagnosing pharyngeal dysphagia and our study cut off value for diagnosing pharyngeal dysphagia

\begin{tabular}{|c|c|c|c|c|c|c|c|c|c|}
\hline Percentage of reduced relative laryngeal movement & TN & $\mathrm{FP}$ & TP & $\mathrm{FN}$ & SN\% & $\mathrm{SP} \%$ & PP\% & PN\% & Eff\% \\
\hline $40 \%$ & 15 & 0 & 9 & 1 & 90.0 & 100 & 100 & 93.8 & 96 \\
\hline $45 \%$ & 15 & 0 & 10 & 0 & 100 & 100 & 100 & 100 & 100 \\
\hline
\end{tabular}

The limitation of this study is that we compare the result of reduced relative laryngeal movement with the clinical result of FEES, rather than the videofluoroscopic examination which is the gold standard examination for the evaluation of swallowing where the laryngeal movement can be readily measured and compared, yet we use the FEES to avoid exposing the children to X-rays, and despite the limitation of this study, the advantage of US of being a noninvasive, radiation-free, and less-expensive bedside procedure in addition to its quantitative value makes it an emergent and promising examination which can be used in the screening and serial monitoring for treatment response of dysphagia in children; another advantage of US examination is that it can be repeated many times without influencing the children or their parent any psychological disturbance.

\section{Conclusion}

Ultrasound can efficiently measure the relative laryngeal movement, which is an important parameter of the swallowing process, and as it gives a numerical value, it can be used as a follow-up bedside test in children suffering from dysphagia.

\section{Abbreviations}

CP: Cerebral palsy; FEES: Fiberoptic endoscopic; US: Ultrasound

\section{Acknowledgements}

Not applicable

\section{Authors' contributions}

AA has performed the ultrasound examination and carried out the interpretation of US images. He has participated in the data collection and writing the manuscript. EA has participated in the data collection, conceived of the study, and helped to draft the manuscript. SB has performed the flexible fiberoptic endoscope procedure and carried out the interpretation of them. HE has participated in the data collection and team organization and participated in the result interpretation. All authors read and approved the final manuscript.

\section{Funding}

No funds, sponsorship, or financial support to be disclosed.

\section{Availability of data and materials}

The datasets used and/or analyzed during the current study are available from the corresponding author on reasonable request.

\section{Ethics approval and consent to participate}

Available

\section{Consent for publication}

Not applicable

\section{Competing interests}

We agree that all copyright ownership is transferable to the Association of University Radiologists when the manuscript is accepted for publication in EJRNM. We certify that the submitted article will not constitute "Redundant Publication." This study is not previously published nor submitted elsewhere and the methods employed respect the Helsinki Declaration of 1975, as revised in 1983. The authors declare that they have no competing interests.

\section{Author details}

${ }^{1}$ Radiology Department, Faculty of Medicine, Ain Shams University, Cairo, Egypt. ${ }^{2}$ Phoniatric Department, Faculty of Medicine, Ain Shams University, Cairo, Egypt. ${ }^{3}$ Physical Medicine Department, Faculty of Medicine, Ain Shams University, Cairo, Egypt.

Received: 22 June 2019 Accepted: 22 July 2019

Published online: 06 September 2019

\section{References}

1. Shkedy Rabani A, Harries N, Namoora I, Al-Jarrah MD et al (2014) Duration and patterns of habitual physical activity in adolescents and young adults with cerebral palsy. Dev Med Child Neurol 56(7):673-680

2. Song J, Park H, Lee $\mathrm{H}$ et al (2015) Effect of neuromuscular electrical stimulation on swallowing functions in children with cerebral palsy: a pilot randomized controlled trial. Hong Kong J Occup Ther 25:1-6

3. Sitton M, Arvedson J, Visotcky A et al (2011) Fiberoptic endoscopic evaluation of swallowing in children: feeding outcomes related to diagnostic groups and endoscopic findings. Int J Pediatr Otorhinolaryngol 75(8):1024-1031

4. Hiss SG, Postma GN (2003) Fiberoptic endoscopic evaluation of swallowing. Laryngoscope 113:1386-1393

5. Huang Y-L, Hsieh S-F, Chang Y-C et al (2009) Ultrasonographic evaluation of hyoid-larynx approximation in dysphagic stroke patients. Ultrasound Med Biol. 35(7):1103-1108

6. Cichero J (2006) Conditions commonly associated with dysphagia. In: Cichero J, Murdoch B (eds) Dysphagia: foundation, theory and practice. Wiley, Chichester, England/New York, pp 237-243

7. Leonard RJ, Kendall KA, McKenzie S et al (2000) Structural displacements in normal swallowing: a videofluoroscopic study. Dysphagia 15:146-152

8. Mendelsohn MS, McConnel FM (1987) Function in the pharyngoesophageal segment. Laryngoscope 97:483-489

9. Sivarao DV, Goyal RK (2000) Functional anatomy and physiology of the upper esophageal sphincter. Am J Med 108(4a):27-37

10. Kim JS, Han ZA, Song DH et al (2013) Characteristics of dysphagia in children with cerebral palsy, related to gross motor function. Am J Phys Med Rehabil. 92(10):912-919

11. Benfer KA, Weir KA, Bell KL et al (2013) Oropharyngeal dysphagia and gross motor skills in children with cerebral palsy. Pediatrics. 131(5):1553-1562

12. Sarwat SA (2016) The role of fiberoptic endoscopic evaluation of swallowing in the assessment of pediatric dysphagia. Egyptian J Otolaryngol. 32:67-74

13. Dodrill P (2015) Gosa MM (2015): Pediatric dysphagia: physiology, assessment, and management. Ann Nutr Metab. 66(5):24-31

14. Chi-Fishman G (2005) Quantitative lingual, pharyngeal and laryngeal ultrasonography in swallowing research: a technical review. Clin Linguist Phon 19:589-604

15. Shawker TH, Sonies B, Hall TE et al (1984) Ultrasound analysis of tongue, hyoid, and larynx activity during swallowing. Invest Radiol 19:82-86

16. Cook IJ, Dodds WJ, Dantas RO et al (1989) Opening mechanisms of the human upper esophageal sphincter. Am J Physiol 257:G748-G759

17. Ishida R, Palmer JB, Hiiemae KM (2002) Hyoid motion during swallowing: factors affecting forward and upward displacement. Dysphagia 17:262-272 
18. Kendall KA, McKenzie S, Leonard RJ et al (2000) Timing of events in normal swallowing: a videofluoroscopic study. Dysphagia 15:74-83

19. Ohmae Y, Logemann JA, Kaiser P et al (1995) Timing of glottic closure during normal swallow. Head Neck 17:394-402

20. Veis SL, Logemann JA (1985) Swallowing disorders in persons with cerebrovascular accident. Arch Phys Med Rehabil 66:372-375

21. Leder SB, Sasaki CT, Burrell MT (1998) Fiberoptic endoscopic evaluation of dysphagia to identify silent aspiration. Dysphagia 13:19-21

22. Kuhl V, Eicke BM, Dieterich M, Urban PP (2003) Sonographic analysis of laryngeal elevation during swallowing. J Neurol 250:333-337

\section{Publisher's Note}

Springer Nature remains neutral with regard to jurisdictional claims in published maps and institutional affiliations.

\section{Submit your manuscript to a SpringerOpen ${ }^{\odot}$ journal and benefit from:}

- Convenient online submission

- Rigorous peer review

- Open access: articles freely available online

High visibility within the field

- Retaining the copyright to your article

Submit your next manuscript at $\boldsymbol{\nabla}$ springeropen.com 